\title{
A Computer Controlled Precision High Pressure Measuring System
}

\author{
S. Sadana ${ }^{1}$, S. Yadav, N. Jha², V. K. Gupta, R. Agarwal ${ }^{1}$, \\ A. K. Bandyopadhyay and T. K. Saxena \\ National Physical Laboratory (NPLI), Council of Scientific and Industrial Research (CSIR), New Delhi - 110012 , India \\ ${ }^{1}$ Electronics and Electrical Engineering Department (EEED), Thapar University, Patiala, Punjab, India \\ ${ }^{2}$ Department of Electronics, University of Delhi South Campus, New Delhi 110 021, India \\ Email: syadav@nplindia.org
}

\begin{abstract}
A microcontroller (AT89C51) based electronics has been designed and developed for high precision calibrator based on Digiquartz pressure transducer (DQPT) for the measurement of high hydrostatic pressure up to 275 MPa. The input signal from DQPT is converted into a square wave form and multiplied through frequency multiplier circuit over 10 times to input frequency. This input frequency is multiplied by a factor of ten using phased lock loop. Octal buffer is used to store the calculated frequency, which in turn is fed to microcontroller AT89C51 interfaced with a liquid crystal display for the display of frequency as well as corresponding pressure in user friendly units. The electronics developed is interfaced with a computer using RS232 for automatic data acquisition, computation and storage. The data is acquired by programming in Visual Basic 6.0. This system is interfaced with the PC to make it a computer controlled system. The system is capable of measuring the frequency up to $4 \mathrm{MHz}$ with a resolution of $0.01 \mathrm{~Hz}$ and the pressure up to $275 \mathrm{MPa}$ with a resolution of $0.001 \mathrm{MPa}$ within measurement uncertainty of $0.025 \%$. The details on the hardware of the pressure measuring system, associated electronics, software and calibration are discussed in this paper.
\end{abstract}

Keywords: Digital pressure calibrator, computer controlled pressure measurements, precision high pressure measuring system, pressure sensor, pressure transducer

\section{INTRODUCTION}

$\mathrm{T}_{\mathrm{s}}^{\mathrm{H}}$ HE LIQUID COLUMN manometers (LCM) and piston gauge manometers (PGM) are often used as primary instruments for realizing the pressure scale in the fundamental units of mass, length, temperature and time through metrological characterization in an independent way without calibration [1]. These mechanical devices provide a direct response to a pressure change, for example, change in the length of the column of mercury or change in the area of piston cylinder assembly. For high pressure applications, PGM are used as reliable primary instruments up to few GPa [1-2]. In other devices and techniques such as mechanical gauges and electronic transducers, the change in the mechanical stresses or change in the resistance of a coil are monitored as function of applied pressure and such instruments are categorized as transfer standard(s) (TS/TSs). Therefore, the use of pressure transducer (PT) with intelligent instrumentation as TS is the convenient way to address such difficulties. The use of PGM as TS is limited up to $500 \mathrm{MPa}$ [3-6] due to their bulky size, high costs, high operation demands and difficulties in transportation.

For the unstandardized values of TSs as a function of pressure change, a suitable correlation is established with primary standards through a calibration process. It is therefore obvious that the accuracy of TSs is limited by the accuracy of primary standard against which these are directly or indirectly calibrated for establishing their own metrological characteristics. The mechanical dial type pressure gauges have recently been used as TSs for the evaluation of participating laboratory's performance through interlaboratory comparison [7-8] in the pressure range up to
70 MPa within the accuracy limit of $+0.25 \%$ of full scale or coarser. The strain gauge type PTs have also been used very sparingly but for high pressure ranges up to 1.4 GPa [9-10]. The capacitance type PT as TS has also been used and produces accurate and repeated results up to $500 \mathrm{MPa}$ [11]. The limited sensitivity, poor linearity and long term stability, zero shifts with time and high hysteresis of the PTs limit their use as a highly precise TS or a pressure measuring device for scientific and industrial applications.

The most recent developments in the field of pressure instrumentation techniques utilize transducers and sensors, based on the principle of detecting pressure induced stress by means of changes in the oscillating frequency of crystalline Quartz resonators. Such PTs effectively address the abovementioned problems and are found to be more reliable with a promising future for accurate and precise pressure measurements [12-13]. One of the features of such PTs is that they can be designed with extremely sharp resonance frequencies with the help of quality oscillator circuitry and hence, it is possible to detect very small changes in the resonant frequencies as a function of applied pressure. One of such PTs has successfully been used as TS in the recently concluded key comparison exercise [14]. Still a major difficulty for use of these devices is their high cost and complex electronics and controls. The authors have recently taken up the work on the research and development of an indigenous low cost, easy to use, precise and accurate intelligent digital pressure calibrator cum TS for the measurement of high hydrostatic pressure up to $280 \mathrm{MPa}$, both in the absolute and gauge pressure modes. The present paper reports the design and development of an indigenous low cost, easy to use, precise and accurate intelligent digital 
pressure calibrator cum transfer standard for the measurement of high hydrostatic pressure up to $280 \mathrm{MPa}$, both in the absolute and gauge pressure modes.

\section{HARDWARE AND ELECTRONICS}

The high precision pressure calibrator thus developed consists of a hydraulic screw pump of volume $5 \mathrm{~cm}^{3}$, an oil reservoir of capacity $150 \mathrm{~cm}^{3}$, two needle valves, a DPQT pressure sensor, power supply, and an output port for gauge under calibration. All the components are mounted on a rigid stainless steel base plate of $2.5 \mathrm{~mm}$ thickness. The whole assembly is then housed in a metallic box. A pictorial view of the calibrator is depicted in Fig.1. The dotted double lines show the metallic pressure transmitting circuit while single lines represent the electronic wiring.

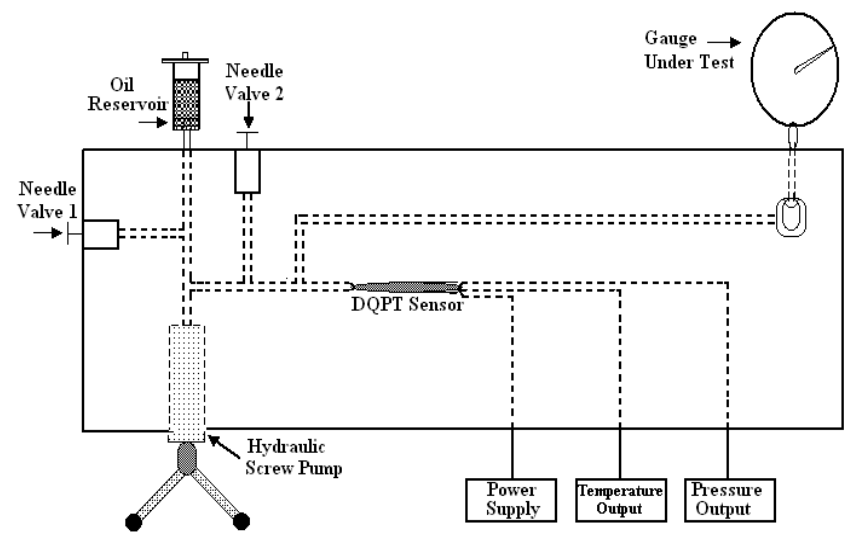

Fig.1. Schematic view of the assembly of precision high pressure measuring system

In the present work, a commercially available DQPT, model $440 \mathrm{~K}$, made by Paroscientific INC, USA is used as sensing element. The associated electronics is designed and developed indigenously with suitable software. The DQPT is connected with a special digital interface board to produce an intelligent transmitter. The PT provides two continuous frequency output signals, one corresponding to the pressure and the other to the sensor's internal temperature. The electronic circuitry of the precision high pressure monitoring instrument has been developed around Atmel's AT89C51 microcontroller. The block diagram of the electronic circuitry is shown in Fig.2.

In this circuit, first of all, the incoming signal is converted into the square wave form with the help of an operational amplifier. Then this signal is fed to the frequency multiplier. The output signal from the multiplier is ten times the input frequency. This signal is then fed to the 24-bit frequency counter through the AND gate. At the other pin of the AND gate, a timing pulse is fed. During this pulse the frequency counter counts the frequency and thereafter the microcontroller reads the frequency from the frequency counter and then after calculations it sends the results to the LCD as well as to the PC through the RS232C interface.

The details of the working principle for such a sensing element and its mechanism are available in the literature $[12-13,16]$. The nominal frequency of pressure resonator is
$38 \mathrm{kHz}$, which changes $10 \%$ as the applied pressure reaches the full scale of $275 \mathrm{MPa}$, while the nominal frequency of the temperature resonator of $172 \mathrm{kHz}$ changes about $50 \times 10^{-6} /{ }^{0} \mathrm{C}$. The temperature compensated pressure output is computed using the following equations derived from the basic physical principle of fixed vibrating beam [12-13].

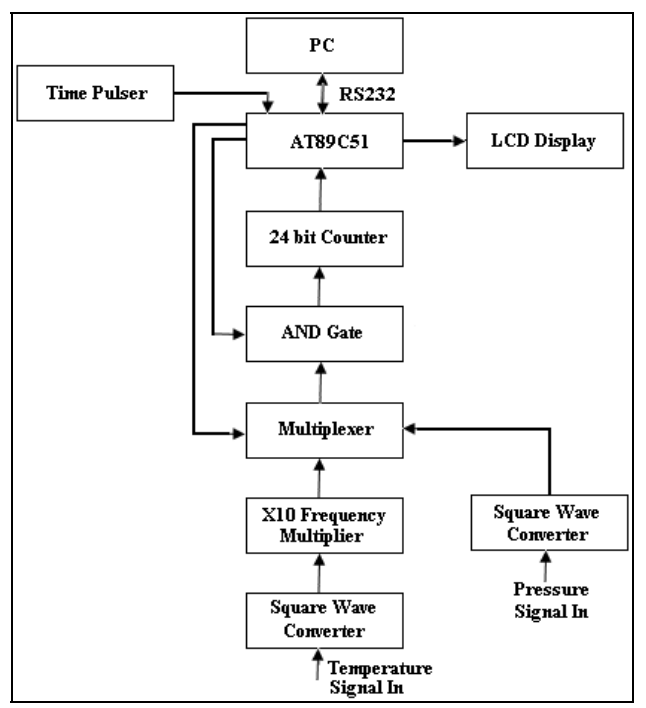

Fig.2. Block diagram of the developed electronics and software

$$
P=C\left(1-\frac{T_{0}^{2}}{T^{2}}\right)\left[1-D\left(1-\frac{T_{0}^{2}}{T^{2}}\right)\right]
$$

where $C$ and $D$ are calibration coefficients, $T_{0}$ is the output time period (reciprocal of frequency output) at zero pressure and $T$ the output time period at applied pressure. If $U(t)$ and $U_{0}$ are the output time periods in microseconds for temperatures at $t$ and zero degree Celsius, respectively, then the temperature measured by the resonator in degree Celsius is computed using:

where

$$
T\left({ }^{\circ} \mathrm{C}\right)=Y_{1} U+Y_{2} U^{2}+Y_{3} U^{3}
$$

$$
U=U(t)-U_{0}
$$

In order to calculate the values of $C, D$ and $T_{0}$, the value of $U$ is used and these coefficients are computed using the following equations;

$$
\begin{gathered}
C=C_{1}+C_{2} U+C_{3} U^{2} \\
D=D_{1}+D_{2} U \\
T_{0}=T_{1}+T_{2} U+T_{3} U^{2}+T_{4} U^{3}
\end{gathered}
$$

All the values of the coefficients obtained from the initial calibration are: $U_{0}=5.874551 \mu \mathrm{s}, Y_{1}=-3883.580{ }^{\circ} \mathrm{C} / \mu \mathrm{s}, Y_{2}$ $=-10551.09{ }^{\circ} \mathrm{C} / \mu \mathrm{s}, Y_{3}=0, C_{1}=-2821.27 \mathrm{MPa}, C_{2}=-$ $2377.55 \mathrm{MPa} / \mu \mathrm{s}, C_{3}=121489.29 \mathrm{MPa} / \mu \mathrm{s}, D_{1}=0.015656$, $D_{2}=0, T_{1}=30.32175 \mu \mathrm{s}, T_{2}=0.776035 \mu \mathrm{s} / \mu \mathrm{s}, T_{3}=$ $66.07862 \mu \mathrm{s} / \mu \mathrm{s}^{2}$ and $T_{4}=0$. 


\section{SOFTWARE}

The program for AT89C51 has been written in 8051 assembly language. The program first selects the pressure gauge frequency and takes the data from the frequency counter at regular intervals of 10 seconds by gating the signal for the defined period and stores the same in the RAM. Thereafter, it switches the multiplexer to temperature gauge data and again takes the frequency of temperature gauge. After taking the data in binary form, the program sends the data to the PC through RS232C interface.

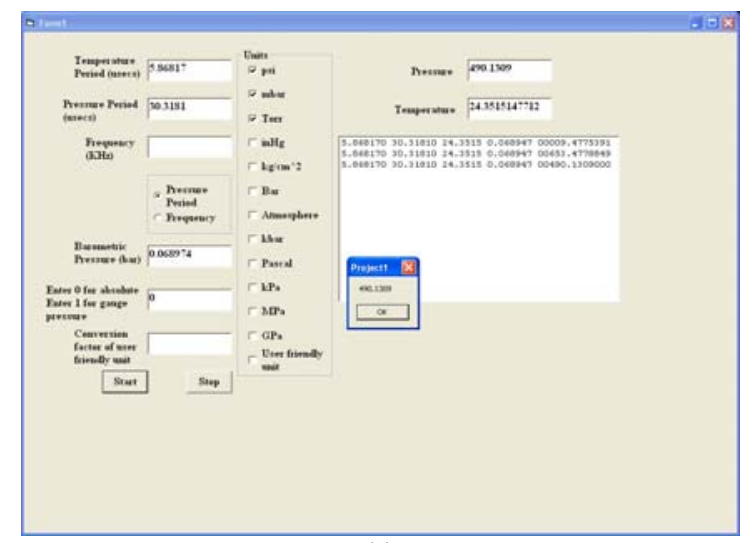

(a)

\begin{tabular}{|c|c|c|c|c|c|}
\hline Desult.txt - & Notepad & & & 口回 & \\
\hline File Edit Format & View Help & & & & \\
\hline $\mathrm{x}$ & $\mathrm{T}$ & t & patom & peorr (A) & 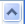 \\
\hline 5.86804 & 30.31844 & 24.83869574 & 0.98594 & 0.935783054 & \\
\hline 5.868245 & 30.3187 & 24.07028468 & 0.98233 & 0.693091614 & \\
\hline 5.8681 & 30.31834 & 24.61388674 & 0.98173 & 1.01993326 & \\
\hline 5.86822 & 30.31859 & 24.16404082 & 0.98539 & 0.791644569 & \\
\hline 5.86822 & 30.31859 & 24.16404082 & 0.98536 & 0.791644569 & \\
\hline 5.868325 & 30.31841 & 23.77017638 & 0.98157 & 0.945812924 & \\
\hline 5.867975 & 30.03994 & 25.08215311 & 0.98589 & 250.6679953 & \\
\hline 5.868245 & 30.04051 & 24.07028468 & 0.98237 & 250.1407824 & \\
\hline 5.867775 & 30.03934 & 25.83069348 & 0.9817 & 251.2256951 & \\
\hline 5.86822 & 30.03944 & 24.16404082 & 0.9857 & 251.1143733 & \\
\hline 5.868155 & 30.0402 & 24.40774508 & 0.98515 & 250.4247059 & \\
\hline 5.8684 & 30.04048 & 23.48870219 & 0.98175 & 250.1668375 & \\
\hline 5.867985 & 29.7696 & 25.04470393 & 0.98572 & 499.9575331 & \\
\hline 5.868235 & 29.76999 & 24.10778872 & 0.98239 & 499.5931058 & \\
\hline 5.867735 & 29.76949 & 25.98030026 & 0.98163 & 500.0674682 & \\
\hline 5.868195 & 29.76991 & 24.25778378 & 0.98575 & 499.6673791 & \\
\hline 5.86816 & 29.76978 & 24.38900176 & 0.98491 & 499.788621 & \\
\hline 5.868395 & 29.77 & 23.50747083 & 0.98173 & 499.587728 & \\
\hline 5.86801 & 29.50647 & 24.95107176 & 0.98551 & 749.3543165 & \\
\hline 5.868225 & 29.50676 & 24.14529065 & 0.98251 & 749.083289 & \\
\hline 5.867735 & 29.50628 & 25.98030026 & 0.9816 & 749.3550232 & \\
\hline 5.868175 & 29.50663 & 24.33276865 & 0.98601 & 749.2059414 & \\
\hline 5.868185 & 29.5066 & 24.29527727 & 0.98471 & 749.2351919 & \\
\hline 5.86839 & 29.5068 & 23.52623894 & 0.98183 & 749.0543575 & \\
\hline
\end{tabular}

(b)

Fig.3. (a) Form window in Visual Basic and (b): Output file where $X$ and $T$ are the time periods for temperature and pressure outputs, $t$ is the calculated measurement temperature, patom is the measured atmospheric pressure and pcorr (A) represents the corrected measured absolute pressure

The computer program, which is written in Visual BASIC6.0 language to run on Windows XP platform, converts the raw data into pressure using (1)-(6) and the window project form thus created is shown in Fig.3(a). The data is then sent back in ASCII format to the LCD for display. It also stores the data in the user defined file as well as displaying them on the PC screen. The calculated data related to temperature period, pressure period, temperature, barometric pressure and absolute pressure is stored in the user defined output file as shown in Fig.3(b). The final data is used to generate calibration report of the pressure gauge under test. The resolution of the pressure displayed on the LCD is $0.001 \mathrm{MPa}$.

\section{CALIBRATION OF DQPT}

The calibration and traceability of DQPT is performed by comparison method against NPL500MPA, a national hydraulic secondary pressure standard, using the similar calibration procedure as reported earlier [13, 17]. The calibration was performed at 12 equally spaced pressure points in increasing as well as decreasing order of pressures to accommodate the effect of hysteresis and thus making the total number of 24 observations in one pressure cycle. After reaching full-scale pressure in increasing order of pressure, 10 minutes were allowed to pass before repeating the observations in decreasing order of pressure. The pressure transmitting fluid used in the present work is di (2ethylhexyl) sebacate oil, commercially known as BIS or DOS.

Sufficient time of at least 10-15 minutes was given between two successive observations to allow the system to reach the thermal equilibrium state. Three pressure cycles were employed so that the minimum number of observations at each pressure point was 6 and there were 72 observations in total as a whole. The measurement uncertainty is estimated as per methodology reported earlier [19]. The calibration factor $\left(C_{\mathrm{f}}\right)$ of the DQPT is computed using;

$$
C_{\mathrm{f}}=\frac{p_{\mathrm{STD}}}{p_{\mathrm{DQPT}}}
$$

where $p_{\mathrm{STD}}$ and $p_{\mathrm{DQPT}}$ are the measured pressure by the standard and the DQPT, respectively.

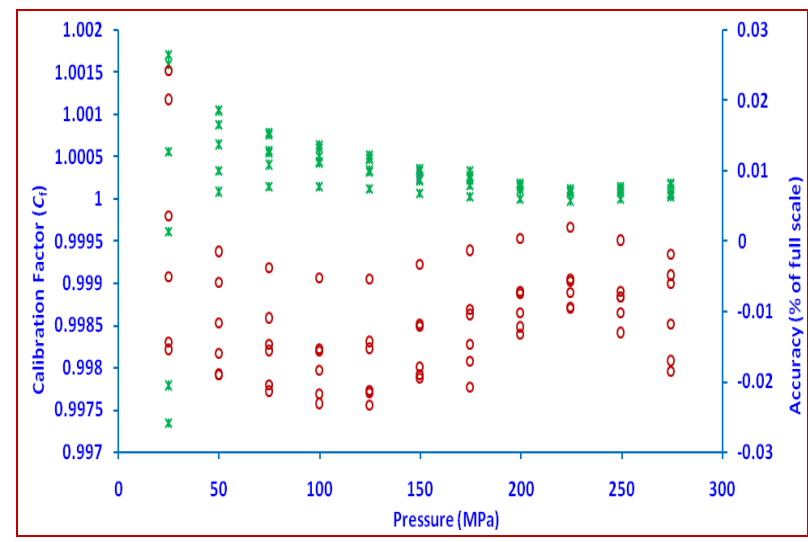

Fig.4. Calibration factor $(*)$ and full scale accuracy (o) as a function of pressure are shown as primary and secondary $\mathrm{x}$-axis, respectively.

The calibration factor as a function of applied pressure is shown in Fig.4 along with full scale accuracy. The full scale accuracy is determined from the difference of $p_{\text {STD }}$ and $p_{\mathrm{DQPT}}$ as follows:

$$
\operatorname{Accuracy}(\%)=\left\{\frac{\left(p_{\mathrm{DQPT}}-p_{\mathrm{STD}}\right)}{p_{\mathrm{FSP}}}\right\} \cdot 100
$$

where $p_{\text {FSP }}$ is the full scale pressure (275 MPa in the present case) measured by the DQPT. The mean calibration factor or 
the best fit line can be used to compute the equivalent pressure as reported elsewhere [18]. The full scale accuracy is found to be well within $+0.015 \%$ except initial pressure point of $25 \mathrm{MPa}$ where accuracy varies up to $\pm 0.025 \%$ but still within the manufacturer specified accuracy of the sensing element of $\pm 0.025 \%$. The maximum accuracy of calibrator of $\pm 0.025 \%$ at $25 \mathrm{MPa}$ is obvious at less than $10 \%$ of the full scale pressure $[16,18]$.

\section{CONCLUSIONS}

Suitable electronics has been designed and developed for the digitization of high DQPT that can measure pressure up to $275 \mathrm{MPa}$. The electronics developed is very compact, noise free, cost efficient and sagaciously designed. The intelligent electronics developed measures frequency up to 4 $\mathrm{MHz}$ with a fine resolution of $0.01 \mathrm{~Hz}$. The frequency obtained is converted into pressure using equations derived from the basic physical principle of fixed vibrating beam. The calibration of DQPT is carried out against national secondary pressure standard. The calibration results obtained are well within the manufacturer specified accuracy. It is evident from the study that this PT can be used as a reliable transfer pressure standard for the accurate and precise hydrostatic pressure measurements and for the calibration of pressure measuring instruments having accuracy of $\pm 0.025 \%$ or coarser in the pressure range from atmospheric pressure to $275 \mathrm{MPa}$. Excellent linearity, repeatability and high resolution in the pressure values are obtained using the present precision electronics. The electronics developed is interfaced with a computer using RS232 for automatic data acquisition, computation and storage by programming in Visual Basic 6.0 which enables us to display both frequency and pressure simultaneously on a LCD. Although the requirement for the measurement of frequency for this particular transducer was in the range of $30-40 \mathrm{kHz}$ for pressure and up to $170 \mathrm{kHz}$ for temperature, the intelligent electronics developed has now made it possible to measure the frequency up to $4 \mathrm{MHz}$, which implies that the electronics can be used with pressure transducers of both higher and lower ranges. The frequency counter has a very fine resolution of $0.01 \mathrm{~Hz}$. The electronics developed along with the associated software has made the complete system user friendly. The automated system will be of great help in reducing the time required for calibration and also the physical work.

For the pressure generation, generally, manual screw type mechanical hydraulic pump is used. This is very tedious, time consuming, cumbersome and involves heavy physical work. It is possible to drive a mechanical screw type piston pump mechanism through a stepping servomotor with the help of suitable mechanical arrangements, electronic circuitry, controls and appropriate software. All the mechanical, electronics and control systems can be embedded into a single unit which can be used as digital pressure calibrator for the calibration of pressure measuring instruments in the pressure range up to $275 \mathrm{MPa}$, both in absolute and gauge mode. The calibrator can also be used as transfer standards for the intercomparison and proficiency testing exercises.

\section{REFERENCES}

[1] Heydemann, P.L.M., Welch, W.E. (1975). Piston gauges. In Le Neindre, B., Voder, B. (eds.) Experimental Thermodynamics. London: Butterworths, 147-201.

[2] Yadav, S., Prakash, O., Gupta, V.K., Bandyopadhyay, A.K. (2007). The effect of pressure-transmitting fluids in the characterization of a controlled clearance piston gauge up to $1 \mathrm{GPa}$. Metrologia, 44, 222-233.

[3] Sabuga, W., Bergogilo, M., Rabault, T., Waller, B., Torres, J.C., Olson, D.A., Agarwal, A., Kobata, T., Bandyopadhyay, A.K. (2005). Final report on Key comparison CCM.P-K7 in the range $10 \mathrm{MPa}$ to 100 MPa of hydraulic gauge pressure. Metrologia (Tech. Suppl.), 42, 07005.

[4] Driver, R.G., Olson, D.A., Yadav, S., Bandyopadhyay, A.K. (2006). Final report on APMP.SIM.M.P-K7: Bilateral comparison between NIST (USA) and NPLI (India) in the hydraulic pressure region $40 \mathrm{MPa}$ to 200 MPa. Metrologia, 43, 07003.

[5] Yadav, S., Bandyopadhyay, A.K., Dilawar, N., Gupta, A.C. (2002). Intercomparison of national hydraulic pressure standards up to $500 \mathrm{MPa}$. Measurement + Control, 35, 47-51.

[6] Sabuga, W., Olson, D.A., Torres, J.C., Yadav, S., Jin, Y., Otal, P., Kobata, T. (2011). CCM key comparison for $500 \mathrm{MPa}$ range of hydraulic gauge pressure (CCM.P-K13). In $5^{\text {th }}$ CCM International Conference on Pressure Metrology (CCMP5), 2-5 May 2011, Berlin, Germany.

[7] Yadav, S., Gupta, V.K., Prakash, O., Bandyopadhyay, A.K. (2005). Proficiency testing through interlaboratory comparison in the pressure range up to $70 \mathrm{MPa}$ using pressure dial gauge as an artifact. Journal of Scientific and Industrial Research, 64 (10), 722-740.

[8] Yadav, S., Prakash, O., Gupta, V.K., Kumaraswamy, B.V., Bandyopadhyay, A.K. (2008). Evaluation of interlaboratory performance through proficiency testing using pressure dial gauge in the hydraulic pressure measurement up to $70 \mathrm{MPa}$. MAPAN Journal of Metrology Society of India, 23 (2), 79-99.

[9] Birks, A.W. (1981). Strain gauge pressure transducer. Belfast: Queen's University of Belfast. (Internal Report No. 1566)

[10] Kobata, T., Ide, K., Kajikawa, H., Sabuga, W., Scheppner, S., Schultz, W. (2010). Final report on supplementary comparison APMP.M.P-S8 in hydraulic gauge pressure from $100 \mathrm{MPa}$ to $1000 \mathrm{MPa}$. Metrologia, 47, 07009.

[11] Kumar, Y., Kumar, V., Jain, K.K., Kashyap, S.C. (1999). A capacitive pressure gauge as a reliable transfer pressure standards. Sensors and Actuators, B55, 217-221.

[12] Juanarena, D.W., Keeter, S., Albert, W.C. A monolithic quartz resonator pressure transducer for high accuracy and stability pressure measurement. Application note P0001. www.pressuresystems.com. 
[13] Yadav, S., Bandyopadhyay, A.K., Gupta, V.K., Gupta, A.C. (2001). A reliable quartz digital transfer pressure standard for high pressure measurements up to 275 MPa. Journal of the Instrument Society of India, 30 (1), 43-51.

[14] Kobata, T., Bandyopadhyay, A.K., Moore, K., Eltawil, A.A.E., Woo, S.Y., Chan, T.K., Jian, W., Man, J., Con, N.N., Fatt, C.S., Permana, W., Aldammd, M., Sabuga, W., Changpan, T., Hung, C.C., Pengcheng, Z. (2005). Final report on Key comparison APMP.M.P-K7 in hydraulic gauge pressure from $10 \mathrm{MPa}$ to $100 \mathrm{MPa}$. Metrologia (Tech. Suppl.), 42, 07006.

[15] Busse, D.W. (1987). Quartz transducers for precision under pressure. Mechanical Engineering, 109 (5).
[16] Yadav, S., Vijayakumar, D.A., Bandyopadhyay, A.K., Gupta, A.C. (2000). A proposal for the establishment of measurement uncertainty of pressure dial gauges and transducers. Cal Lab: The International Journal of Metrology, 6 (2).

[17] Yadav, S., Gupta, V.K., Prakash, O., Bandyopadhyay, A.K. (2005). Evaluation of associated uncertainties in calibration of direct pressure indicating electromechanical devices. Measurement Science Review, 5, 104-114.

[18] Yadav, S., Gupta, V.K., Bandyopadhyay, A.K. (2010). Investigations on measurement uncertainty and stability of pressure dial gauges and transducers. Measurement Science Review, 10 (4), 130-135.

Received April 15, 2011. Accepted December 12, 2011. 